\title{
NUMERICAL STABILITY OF BLOCK TOEPLITZ ALGORITHMS IN POLYNOMIAL MATRIX COMPUTATIONS
}

\author{
Juan Carlos Zúñiga* Didier Henrion ${ }^{*, * *}$
}

\author{
* LAAS-CNRS \\ 7 Avenue du Colonel Roche, 31077 Toulouse, France \\ ** Department of Control Engineering, Faculty of Electrical \\ Engineering, Czech Technical University in Prague \\ Technická 2, 16627 Prague, Czech Republic
}

\begin{abstract}
We study the problem of computing the eigenstructure of a polynomial matrix. Via a backward error analysis we analyze the stability of some block Toeplitz algorithms to obtain this eigenstructure. We also elaborate on the nature of the problem, i.e. conditioning and posedness.
\end{abstract}

Keywords: Polynomial matrices, eigenstructure, numerical linear algebra, numerical analysis.

\section{INTRODUCTION}

Polynomials and polynomial matrices arise naturally in linear systems. Polynomial matrices represent systems of differential equations or system transfer functions in the complex variable [Kailath, 1980]. Several control problems, even non-linear control problems, can be reduced to the solution of a polynomial matrix equation or Diophantine equation [Kučera, 1993].

Unfortunately, the development and analysis of computational algorithms dealing with polynomials and polynomial matrices is not enough developed. There still exist several gaps to fill up. One of these gaps concerns numerical stability. Polynomials methods are often related with elementary polynomial operations like the Euclidian division, which are known to be numerically unstable, see for example [Gantmacher, 1959].

During the last years, a new class of reliable polynomial algorithms have been developed, see for example [Henrion and Šebek, 1999]. Glob- ally speaking, these modern algorithms are based only on reliable numerical linear algebra methods and avoid elementary operations over polynomials. The common procedure of these modern polynomial algorithms is:

(1) Reformulate the original polynomial problem (OPP) into an equivalent numerical problem (ENP) which can be solved via a numerical linear algebra method.

(2) Translate the numerical solution of the ENP into the solution of the OPP.

When the problem is the computation of structural properties of an $m \times n$ polynomial matrix

$$
A(s)=A_{d} s^{d}+\cdots+A_{1} s+A_{0},
$$

a classical approach to perform step (1) consists in linearizing the polynomial matrix, namely, constructing a pencil matrix $F(s)=F_{1} s+F_{0}$ containing the same structural properties as $A(s)$ [Van Dooren and Dewilde, 1983]. For example, the most accepted method to solve the polynomial eigenvalue problem of $A(s)$ consists in solving the equivalent generalized eigenvalue problem on pen- 
cil $F(s)$ via the QZ algorithm [Moler and Stewart, 1973]. Another approach to achieve (1) consists in constructing block Toeplitz matrices (Sylvester matrices) associated with $A(s)$ using the coefficients $A_{i}$ [Kailath, 1980, Stefanidis et al., 1992].

Fundamentals characteristics of an ENP are algorithmic complexity and numerical stability. Ideally, we also require that the solution of the OPP is easily obtained from the solution of the ENP. In our above example, the exact finite zeros of $F(s)$, which are the exact finite zeros of $A(s)$, can be obtained via the QZ algorithm which is a polynomial time backward stable method [Golub and Van Loan, 1996].

The algorithmic complexity is the total number of elementary operations (flops) executed by an algorithm. The number of flops required to solve our OPP is then the number of flops required by step (1) plus the number of flops required by step (2). ${ }^{1}$

On the other hand, the analysis of accuracy and stability is much more complicated. In general, we require, at least, that the numerical method used to solve the ENP is backward stable. Nevertheless, this requirement is not always sufficient to ensure the reliability of the solution of the OPP. For example, consider again the polynomial eigenvalue problem. In [Tisseur and Meerbergen, 2001] it is showed that the small backward errors in coefficients $F_{1}$ and $F_{0}$ produced by the QZ algorithm can lead to large backward errors in the coefficients $A_{i}$. In words this means that the accurately computed finite zeros of $F(s)$ could not correspond to those of $A(s)$. In conclusion, some questions that have to be answered are:

- does an accurate solution of the ENP imply an accurate solution of the OPP?

- how the errors (backward and forward errors) in the ENP are reflected in the OPP?

In the present paper we give some elements to answer these questions. In particular we analyze the stability and accuracy of some block Toeplitz algorithms to obtain different structural properties of a polynomial matrix. We developed these algorithms in our previous work [Zúñiga and Henrion, 2004a]. There we showed that the Toeplitz approach results in algorithms which perform, in general, faster than the algorithms using linearizations of the polynomial matrix (pencil algorithms). Our aim now is to learn more about the stability and accuracy of these Toeplitz algorithms.

\footnotetext{
1 Sometimes the execution time required by step (2) can be larger than the time required by step (1). See for example the pencil algorithm [Beelen and Veltkamp, 1987] to obtain the null-space structure of a polynomial matrix.
}

After some preliminaries on the stability and accuracy of rank revealing numerical methods, in section 3 we analyze the problem of obtaining the infinite and null-space structure of a polynomial matrix. In section 4 we analyze the computation of the finite structure. Finally, in section 5 we give some general conclusions and we state the lines that future works could follow.

Notations: real $\epsilon$ represents the machine precision in the floating-point arithmetic system, and $O(\epsilon)$ is any constant depending on $\epsilon$, of the same order of magnitude.

\section{PRELIMINARIES}

As we show in [Zúñiga and Henrion, 2004a], finding the eigenstructure of a polynomial matrix $A(s)$ is equivalent to obtaining the null-spaces of some constant block Toeplitz matrices. In other words, our ENP is the computation of the rank and the null-space of a constant $k \times l$ rank-deficient matrix $M$. In this section we analyze the stability and accuracy of some numerical methods for this ENP.

The classical rank revealing methods are the singular value decomposition (SVD) and the LQ dual to (QR) factorization with row (or column) pivoting. Numerical properties of this methods are well known [Golub and Van Loan, 1996, Higham, 2002]. We summarize these properties as follows.

Lemma 1. The computed singular values of a matrix $M$ are the exact ones of a slightly perturbed matrix $M+\Delta$ with $\|\Delta\|_{2} \leq O(\epsilon)\|M\|_{2}$. If $\sigma_{i}$ are the exact singular values of $M$, then

$$
\left|\sigma_{i}-\hat{\sigma}_{i}\right| \leq O(\epsilon)\|M\|_{2} .
$$

Lemma 2. Let the exact LQ factorization of matrix $M$ be $M Q=L$. The computed factors $\hat{Q}$ and $\hat{L}$ satisfy $(M+\Delta) \hat{Q}=\hat{L}$ with $\|\Delta\|_{2} \leq O(\epsilon)\|M\|_{2}$.

Lemma 3. (Higham, 1990). Let the exact LQ factorization of matrix $M$ be $M Q=L$. If we partition $L$ as follows

$$
L=\left[\begin{array}{cc}
L_{11} & 0 \\
L_{21} & L_{22}
\end{array}\right]
$$

where $L_{11}$ is $r \times r$ with non zero elements along the diagonal, then (to first order),

$$
\frac{\left\|\hat{L}_{22}-L_{22}\right\|_{2}}{\|M\|_{2}} \leq \frac{\|\Delta\|_{2}}{\|M\|_{2}}\left(1+\left\|L_{11}^{-1} L_{21}^{T}\right\|_{2}\right)
$$

where $\left\|L_{11}^{-1} L_{21}^{T}\right\|_{2}$, the conditioning of the LQ factorization for rank-deficient matrices, is usually small.

So, from equations (1) and (2) we can expect to recover the rank $r$ of $M$ by applying the SVD and 
counting the number of computed singular values less than or equal to $k\|M\|_{2}$. Alternatively, we can apply the LQ factorization and count the number of columns of $\hat{L}_{22}$ such that $\left\|\hat{L}_{22}\right\|_{2} \leq k\|M\|_{2}$ where $k$ is a tolerance depending on $\epsilon$. Hence, rank determination is not only a problem in matrix computations, but an exercise of interpretation of the numerical results. Moreover, it is wellknown that the problem of detecting whether $\operatorname{rank} M=r$ is ill-posed or $\infty$-conditioned when $r$ is less than the row or column dimension of $M$. As an important implication, the problem of finding the right null-space of a constant matrix $M$ can be well-conditioned only if $M$ has full row-rank. As we will see in the next sections this rank property is hardly verified for the analyzed Toeplitz matrices.

\section{THE INFINITE AND NULL-SPACE STRUCTURES}

All the algorithms analyzed here are developed in [Zúñiga and Henrion, 2004a] and the references mentioned there. Here we are interested in their stability and accuracy. We define the eigenstructure of a polynomial matrix $A(s)$ as the set of its finite zeros and associated eigenvectors, the set of zeros at infinity and associated eigenvectors and the set of polynomial vectors forming a basis of the left and right null-spaces. Another structural information which is related with the eigenstructure is the rank of $A(s)$. In [Zúñiga and Henrion, 2004a] we also show that this rank can be obtained as a sub-product of the process of obtaining the eigenstructure. So, in the remainder of the paper we simply consider that $\rho=\operatorname{rank} A(s)$ is known.

The infinite structure of $A(s)$ is equivalent to the finite structure at $s=0$ of the dual matrix

$$
A_{\text {dual }}(s)=A_{d}+A_{d-1} s+\cdots+A_{0} s^{d} .
$$

If $s=0$ has algebraic multiplicity $m_{A}$ and geometric multiplicity $m_{G}$, then there exists a series of integers $k_{i}>0$ for $i=1,2, \ldots, m_{G}$ such that $m_{A}=$ $k_{1}+k_{2}+\cdots+k_{m_{G}}$ and a series of eigenvectors at infinity $v_{i 1}, v_{i 2}, \ldots, v_{i k_{i}}$ for $i=1,2, \ldots, m_{G}$ such that

$$
\begin{array}{r}
{\left[\begin{array}{ccc}
A_{d} & & \\
\vdots & \ddots & \\
A_{d-k_{i}+1} & \cdots & A_{d}
\end{array}\right]\left[\begin{array}{c}
v_{i 1} \\
\vdots \\
v_{i k_{i}}
\end{array}\right]=} \\
\\
T_{I}\left[A(s), k_{i}\right] \mathcal{V}_{k_{i}}=0
\end{array}
$$

with $v_{11}, v_{21}, \ldots, v_{m_{G} 1}$ linearly independent.

A basis of the null-space of $A(s)$ contains the $n-\rho$ non-zero polynomial vectors $z(s)$ such that $A(s) z(s)=0$. This last equation can be rewritten as follows:

$$
\begin{aligned}
& {\left[\begin{array}{ccc}
A_{d} & & \\
\vdots & \ddots & \\
A_{0} & & A_{d} \\
& \ddots & \vdots \\
& & A_{0}
\end{array}\right]\left[\begin{array}{c}
z_{d_{z}} \\
\vdots \\
z_{1} \\
z_{0}
\end{array}\right]=} \\
& T_{N}\left[A(s), d_{z}+1\right] \mathcal{Z}_{d_{z}}=0 .
\end{aligned}
$$

From Equations (3) and (4) we can derive the ENP associated to the OPP of obtaining the infinite structure and the null-space structure respectively. In [Zúñiga and Henrion, 2004a] we show how indices $k_{i}$ and $d_{z}$ are determined by obtaining the ranks of Toeplitz matrices $T_{I}[A(s), i]$ and $T_{N}[A(s), i]$ of increasing size, for $i=1,2, \ldots$ For example let $r_{1}=\operatorname{rank} T_{I}[A(s), 1]$, if $r_{1}=\rho$ then matrix $A(s)$ has no infinite zeros. If $r_{1}<\rho$, there are $\rho-r_{1}$ chains of at least one infinite eigenvector. On the other hand, if $T_{N}[A(s), i]$ has full rank, then there are no vectors of degree $i-1$ in a minimal basis of the null space of $A(s)$. This dependence on the rank of different matrices makes the problem of obtaining the eigenstructure of $A(s)$ ill-posed or $\infty$-conditioned. In other words, a small perturbation in $A(s)$ could change the number of chains of eigenvectors and their lengths, or the nullity of $A(s)$ and the degree of the vectors in the minimal basis of its null-space.

In principle, we cannot control the conditioning or posedness of a problem, but we can ensure, at least, that the computed solution has a small backward error. Notice that

$$
T_{N}[A(s), i]=\left[\begin{array}{ccc}
\multicolumn{3}{c}{T_{I}[A(s), i]} \\
A_{d-i} & & \\
\vdots & \ddots & \\
A_{0} & & A_{d-i} \\
& \ddots & \vdots \\
& & A_{0}
\end{array}\right]
$$

so, in the remainder of this section we only analyze the backward error produced when computing the null-space structure of $A(s) .{ }^{2}$ The result we expect is that the computed null-space vector $\hat{z}(s)=\hat{z}_{0}+\hat{z}_{1} s+\cdots+\hat{z}_{d_{z}} s^{d_{z}}$ is the exact nullspace vector of a slightly perturbed polynomial matrix

$$
\begin{array}{r}
A(s)+\Delta(s)=\left(A_{0}+\Delta_{0}\right)+\left(A_{1}+\Delta_{1}\right) s+\cdots \\
+\left(A_{d}+\Delta_{d}\right) s^{d}
\end{array}
$$

where $\|\Delta(s)\|$ is small for some polynomial matrix norm. Ideally we want

$$
\left\|\Delta_{i}\right\| \leq O(\epsilon)\left\|A_{i}\right\|_{2} .
$$

\footnotetext{
2 The obtained results can be directly applied to the infinite structure.
} 
Equivalently, in terms of the ENP, what we want is that $\left(T_{N}\left[A(s), d_{z}+1\right]+T_{N}\left[\Delta(s), d_{z}+1\right]\right) \hat{\mathcal{Z}}_{d_{z}}=0$ where

$$
\begin{gathered}
T_{N}\left[\Delta(s), d_{z}+1\right]=\left[\begin{array}{ccc}
\Delta_{d} & & \\
\vdots & \ddots & \\
\Delta_{0} & & \Delta_{d} \\
& \ddots & \vdots \\
& & \Delta_{0}
\end{array}\right], \\
\left\|\Delta_{i}\right\| \leq O(\epsilon)\left\|A_{i}\right\|_{2}
\end{gathered}
$$

and where $\hat{\mathcal{Z}}_{d_{z}}$ is the computed null-space of $T_{N}\left[A(s), d_{z}+1\right]$. The folowing result gives a bound for the error $\Delta(s)$ when using the LQ factorization to solve the ENP (4).

Theorem 4. Let $A(s)$ be a polynomial matrix and suppose that it has a vector $z(s)$ of degree $d_{z}$ in the basis of its null-space. The computed vector $\hat{z}(s)$, obtained from (4) via the LQ factorization, is the exact null-space vector of the slightly perturbed matrix $A(s)+\Delta(s)$ with

$$
\left\|\Delta_{i}\right\|_{2} \leq O(\epsilon)\left\|T_{N}\left[A(s), d_{z}+1\right]\right\|_{2} .
$$

PROOF. From Lemma 2, we obtain

$$
\left(T_{N}\left[A(s), d_{z}+1\right]+\Phi\right) \hat{\mathcal{Z}}_{d_{z}}=0
$$

with

$$
\begin{gathered}
\Phi=\left[\begin{array}{ccc}
\Delta_{d}^{0} & & \\
\vdots & \ddots & \\
\Delta_{0}^{0} & & \Delta_{d}^{d_{z}} \\
& \ddots & \vdots \\
\times & & \Delta_{0}^{d_{z}}
\end{array}\right], \\
\|\Phi\|_{2} \leq O(\epsilon)\left\|T_{N}\left[A(s), d_{z}+1\right]\right\|_{2}
\end{gathered}
$$

where $\times$ represents possibly non zero blocks. We can always apply row elementary operations grouped in a left multiplier $P=I+E$ with $\|E\|_{2} \leq O(\epsilon)\left\|T_{N}\left[A(s), d_{z}+1\right]\right\|_{2}$ such that

$$
\begin{array}{r}
P\left(T_{N}\left[A(s), d_{z}+1\right]+\Phi\right) \hat{\mathcal{Z}}_{d_{z}}= \\
\left(T_{N}\left[A(s), d_{z}+1\right]+\right. \\
\left.T_{N}\left[\Delta(s), d_{z}+1\right]\right) \hat{\mathcal{Z}}_{d_{z}}=0 .
\end{array}
$$

Transformation matrix $P$ and thus $T_{N}\left[\Delta(s), d_{z}+\right.$ $1]$ are not unique, nevertheless we can always check that

$$
\left\|T_{N}\left[\Delta(s), d_{z}+1\right]\right\|_{2} \leq O(\epsilon)\left\|T_{N}\left[A(s), d_{z}+1\right]\right\|_{2}
$$

or equivalently

$$
\left\|\Delta_{i}\right\|_{2} \leq O(\epsilon)\left\|T_{N}\left[A(s), d_{z}+1\right]\right\|_{2}
$$

which is the expected result.

This result shows that our Toeplitz algorithm has a bounded backward error. On the other hand, because of the ill-posedness of the problem, the forward error is not bounded. First, the number and the degree of the computed vectors could be different from those corresponding to the exact null-spaces. Second, for a given degree $d_{z}$, the coefficients of the computed vector could be also different from the exact ones because, in general, matrix $T_{N}\left[A(s), d_{z}+1\right]$ has not full row-rank.

\section{THE FINITE STRUCTURE}

Let $\left\{\alpha_{1}, \alpha_{2}, \ldots, \alpha_{q}\right\}$ be the set of finite zeros of $A(s)$, namely, the values such that $\operatorname{rank} A\left(\alpha_{j}\right)<$ $\rho$, for $j=1,2, \ldots, q$. Suppose that $\alpha_{j}$ has algebraic multiplicity $m_{A}$ and geometric multiplicity $m_{G}$, so that there exists a series of integers $k_{i}>0$ for $i=1,2, \ldots, m_{G}$ such that $m_{A}=k_{1}+k_{2}+$ $\cdots+k_{m_{G}}$ and a series of characteristic vectors $u_{i 1}, u_{i 2}, \ldots, u_{i k_{i}}$ for $i=1,2, \ldots, m_{G}$ associated to $\alpha_{j}$ such that

$$
\begin{aligned}
{\left[\begin{array}{cccc}
A^{(0)} & & & 0 \\
A^{(1)} & A^{(0)} & & \\
\vdots & & \ddots & \\
A^{\left(k_{i}-1\right)} & \ldots & A^{(1)} & A^{(0)}
\end{array}\right]\left[\begin{array}{c}
u_{i 1} \\
u_{i 2} \\
\vdots \\
u_{i k_{i}}
\end{array}\right]=} \\
T_{F}\left[A\left(\alpha_{j}\right), k_{i}\right] \mathcal{U}_{k_{i}}=0
\end{aligned}
$$

with $u_{11}, u_{21}, \ldots, u_{m_{G} 1}$ linearly independent and where

$$
A^{(t)}=\frac{1}{t !}\left[\frac{\mathrm{d}^{t} A(s)}{\mathrm{d} s^{t}}\right]_{s=\alpha_{j}} .
$$

Integer $k_{i}$ is the length of the $i$ th chain of characteristic vectors associated to $\alpha_{j}$.

As well as for the infinite and null-space structures, the ENP associated here consists in obtaining the rank and null-spaces of constant Toeplitz matrices $T_{F}\left[A\left(\alpha_{j}\right), i\right]$ of increasing size, for $i=1,2, \ldots$ In [Zúñiga and Henrion, 2004a] we sketch some methods to find integer $k_{i}$. As soon as $k_{i}$ is determined, solving (8) yields the expected vectors. Nevertheless, notice that matrix $T_{F}\left[A\left(\alpha_{j}\right), k_{i}\right]$ is also computed. Moreover, its blocks are the evaluation of $A(s)$ and its derivatives at a value $\alpha_{j}$ which also has to be computed. So, the propagation of the different errors has to be considered.

\subsection{Evaluation of a polynomial matrix}

The standard method for evaluating a polynomial at a given point $\alpha$ is Horner's method which is straightforwardly extended to a polynomial matrix. Horner's method is backward stable.

Lemma 5. (Higham, 2002). Consider the polynomial matrix $A(s)$. The computed value $\hat{A}^{(j)}$ is 
the exact evaluation in $\alpha$ of the $j$ th derivate of the slightly perturbed matrix $A(s)+\Delta(s)$ with $\left\|\Delta_{i}\right\|_{2} \leq O(\epsilon)\left\|A_{i}\right\|_{2}$.

\subsection{The polynomial eigenvalue problem}

We can show that the zeros of a polynomial matrix $A(s)$ correspond to the zeros of a companion pencil $F(s)=s F_{1}+F_{0}$. So, by obtaining the zeros of $F(s)$ with the QZ algorithm, we theoretically obtain the zeros of $A(s)$. The QZ algorithm is backward stable, so the computed zeros are the exact ones of a slightly perturbed pencil $F(s)+$ $\Delta F(s)$ where $\Delta F(s)=s \Delta F_{1}+\Delta F_{0}$. Nevertheless, a small backward error $\Delta F(s)$ can imply a large backward error $\Delta(s)$ in $A(s)$ [Tisseur and Meerbergen, 2001]. In [Edelman and Murakami, 1995] this problem is analyzed with geometrical arguments and an exact first order perturbation expression for the polynomial eigenvalue problem is derived. In [Lemonnier and Van Dooren, 2004] an expression for the polynomial sensitivity $\phi_{\text {pol }}$ under perturbations in $F(s)$ is also derived and an optimal scaling for $F(s)$ is proposed. The goal of the scaling is to minimize $\phi_{\text {pol }}$. Numerical experiments show that the backward error $\Delta(s)$ is suitably small when we solve the polynomial eigenvalue problem via the QZ algorithm over a scaled pencil $D_{2}^{-1} F(s) D_{1}$.

Another strategy to obtain a small backward error $\Delta(s)$ consists in scaling the coefficients $A_{i}$ and the variable $s$, namely, in changing the polynomial basis in which the columns or the rows of $A(s)$ are represented. In our framework, this strategy has more sense that the scaling over the pencil $F(s){ }^{3}$ In [Fan et al., 2003] this type of scaling is proposed for the quadratic eigenvalue problem. For the more general polynomial eigenvalue problem, it is expected that the scaling over the pencil $F(s)$ is equivalent to a change of basis but there are not yet results on the subject.

For our purposes in this paper, we simply consider that, if $\alpha$ is a finite zero of the polynomial matrix $A(s)$, then the computed zero $\bar{\alpha}=\alpha+\Delta \alpha$ is the exact finite zero of $A(s)+\Delta(s)$ with $\left\|\Delta_{i}\right\|_{2} \leq$ $O(\epsilon)\left\|A_{i}\right\|_{2}$.

\subsection{The associated eigenvectors}

Now, we return to the problem of solving Equation (8). The result we expect is that a computed chain of $k$ eigenvectors $\left\{\hat{u}_{1}, \hat{u}_{2}, \ldots, \hat{u}_{k}\right\}$ associated to the

\footnotetext{
3 Notice that block columns of block Toeplitz matrices associated with $A(s)$ are the representation of the columns of $A(s)$ in a canonical polynomial basis $\left\{I s^{d}, \ldots, I s, I\right\}$. So, a change of basis changes directly the properties of the associated block Toeplitz matrices.
}

computed zero $\hat{\alpha}$ is an exact chain of eigenvectors associated to the exact zero $\hat{\alpha}$ of the slightly perturbed matrix $A(s)+\Delta(s)$ with $\|\Delta(s)\|$ small for some polynomial matrix norm. Ideally we want

$$
\left\|\Delta_{i}\right\|_{2} \leq O(\epsilon)\left\|A_{i}\right\|_{2}
$$

Equivalently, in terms of the ENP, we expect that $\left(T_{F}[A(\hat{\alpha}), k]+T_{F}[\Delta(\hat{\alpha}), k]\right) \hat{\mathcal{U}}_{k}=0$ where

$$
\begin{aligned}
& T_{F}[\Delta(\hat{\alpha}), k]= {\left[\begin{array}{ccc}
\Delta^{(0)} & & 0 \\
\vdots & \ddots & \\
\Delta^{(k-1)} & \cdots & \Delta^{(0)}
\end{array}\right], } \\
&\left\|\Delta_{i}\right\|_{2} \leq O(\epsilon)\left\|A_{i}\right\|_{2}
\end{aligned}
$$

and where

$$
\Delta^{(t)}=\frac{1}{t !}\left[\frac{\mathrm{d}^{t} \Delta(s)}{\mathrm{d} s^{t}}\right]_{s=\hat{\alpha}} .
$$

The following result gives a bound for the error $\Delta(s)$ when using the LQ factorization to solve the ENP (8).

Theorem 6 . Let $A(s)$ be a polynomial matrix and suppose that it has a finite zero $\alpha$ and a chain of $k$ associated eigenvectors $\left\{u_{1}, u_{2}, \ldots, u_{k}\right\}$. The computed vectors $\left\{\hat{u}_{1}, \hat{u}_{2}, \ldots, \hat{u}_{k}\right\}$ associated to the computed zero $\hat{\alpha}$, obtained from (8) via the LQ factorization, are the exact vectors associated to the exact finite zero $\hat{\alpha}$ of the slightly perturbed matrix $A(s)+\Delta(s)$ with

$$
\left\|\Delta^{(j)}\right\|_{2} \leq O(\epsilon)\left\|T_{F}[a(|\hat{\alpha}|), k]\right\|_{2},
$$

and where $a(s)=\left\|A_{d}\right\| s^{d}+\cdots+\left\|A_{0}\right\|$.

PROOF. In this proof we use the notation $\gamma_{i}$ to refer to a constant of the same magnitude as $\epsilon$. The computed finite zero $\hat{\alpha}$ is the exact finite zero of matrix $A_{1}(s)=A(s)+\Delta_{1}(s)$ with $\left\|\Delta_{1_{i}}\right\|_{2} \leq \gamma_{1}\left\|A_{i}\right\|_{2}$. Now, applying Horner's method to $A_{1}(s)$, and from Lemma 5 , we obtain that $\hat{A}_{1}^{(j)}=\left(A_{1}(s)+\Delta_{2}(s)\right)^{(j)}$ with $\left\|\Delta_{2_{i}}\right\|_{2} \leq$ $\gamma_{2}\left\|A_{1_{i}}\right\|_{2}$ or equivalently,

$\left\|\Delta_{2_{i}}\right\|_{2} \leq \gamma_{2}\left\|A_{i}\right\|_{2}+\gamma_{2} \gamma_{1}\left\|A_{i}\right\|_{2}=\left(\gamma_{2}+\gamma_{2} \gamma_{1}\right)\left\|A_{i}\right\|_{2}$.

So, $\hat{A}_{1}^{(j)}=\left(A(s)+\Delta_{3}(s)\right)^{(j)}$ with

$\left\|\Delta_{3_{i}}\right\|_{2} \leq \gamma_{1}\left\|A_{i}\right\|_{2}+\left(\gamma_{2}+\gamma_{2} \gamma_{1}\right)\left\|A_{i}\right\|_{2}=\gamma_{3}\left\|A_{i}\right\|_{2}$.

Finally we obtain the computed value of $T_{F}[A(\hat{\alpha}), k]$ related to the computed zero $\hat{\alpha}$

$$
\begin{array}{r}
\hat{T}_{F}[A(\hat{\alpha}), k]=\left[\begin{array}{ccc}
A_{3}^{(0)} & & \\
\vdots & \ddots & \\
A_{3}^{(k-1)} & \cdots & A_{3}^{(0)}
\end{array}\right]= \\
T_{F}[A(\hat{\alpha}), k]+T_{F}\left[\Delta_{3}(\hat{\alpha}), k\right] .
\end{array}
$$


Now, from Lemma 2, and introducing an elementary transformation as in (7), we obtain the backward stable result $\left(\hat{T}_{F}[A(\hat{\alpha}), k]+T_{F}\left[\Delta_{4}(\hat{\alpha}), k\right]\right) \hat{\mathcal{U}}_{k}=$ 0 where

$$
\begin{aligned}
\left\|T_{F}\left[\Delta_{4}(\hat{\alpha}), k\right]\right\|_{2} \leq & \gamma_{4}\left\|T_{F}[A(\hat{\alpha}), k]\right\|_{2}+ \\
& \gamma_{4}\left\|T_{F}\left[\Delta_{3}(\hat{\alpha}), k\right]\right\|_{2} .
\end{aligned}
$$

Since $\left\|\Delta_{3_{i}}\right\|_{2} \leq \gamma_{3}\left\|A_{i}\right\|_{2}$, we can show that

$$
\left\|\Delta_{3}^{(t)}\right\| \leq \gamma_{3} \frac{1}{t !}\left[\frac{\mathrm{d}^{t} a(s)}{\mathrm{d} s^{t}}\right]_{s=|\alpha|}
$$

where $a(s)=\left\|A_{d}\right\| s^{d}+\cdots+\left\|A_{0}\right\|$ and then

$$
\left\|T_{F}\left[\Delta_{3}(\hat{\alpha}), k\right]\right\|_{2} \leq \gamma_{3}\left\|T_{F}[a(|\hat{\alpha}|), k]\right\|_{2} .
$$

Therefore

$$
\begin{array}{r}
\left\|T_{F}\left[\Delta_{4}(\hat{\alpha}), k\right]\right\|_{2} \leq \gamma_{4}\left\|T_{F}[A(\hat{\alpha}), k]\right\|_{2}+ \\
\gamma_{4} \gamma_{3}\left\|T_{F}[a(|\hat{\alpha}|), k]\right\|_{2} .
\end{array}
$$

Finally $\left(T_{F}[A(\hat{\alpha}), k]+T_{F}[\Delta(\hat{\alpha}), k]\right) \hat{\mathcal{U}}_{k}=0$ where

$$
\begin{array}{r}
\left\|T_{F}[\Delta(\hat{\alpha}), k]\right\|_{2} \leq \gamma_{4}\left\|T_{F}[A(\hat{\alpha}), k]\right\|_{2}+ \\
\left(\gamma_{4} \gamma_{3}+\gamma_{3}\right)\left\|T_{F}[a(|\hat{\alpha}|), k]\right\|_{2} .
\end{array}
$$

Now, notice that

$$
\left\|A^{(t)}\right\| \leq \frac{1}{t !}\left[\frac{\mathrm{d}^{t} a(s)}{\mathrm{d} s^{t}}\right]_{s=|\alpha|}
$$

and so

$$
\left\|T_{F}[A(\hat{\alpha}), k]\right\|_{2} \leq\left\|T_{F}[a(|\hat{\alpha}|), k]\right\|_{2} .
$$

Therefore

$\left\|T_{F}[\Delta(\hat{\alpha}), k]\right\|_{2} \leq\left(\gamma_{4}+\gamma_{3}+\gamma_{4} \gamma_{3}\right)\left\|T_{F}[a(|\hat{\alpha}|), k]\right\|_{2}$, or equivalently

$$
\left\|\Delta^{(j)}\right\|_{2} \leq O(\epsilon)\left\|T_{F}[a(|\hat{\alpha}|), k]\right\|_{2},
$$

which is the expected result.

This result shows that the block Toeplitz algorithm has a backward error bound. On the other hand, the forward error is not bounded. Because of the ill-posedness of the problem, the number and the length of the computed chains of vectors could be different from those corresponding to the exact ones. For a given length $k$, the coefficients of the computed vectors could be also different from the exact ones because, in general, matrix $T_{F}[A(\hat{\alpha}), k]$ has not full row-rank.

\section{CONCLUSIONS AND PERSPECTIVES}

The results presented here are only preliminary, nevertheless they show that rigorous numerical linear algebra analysis can also be applied to the block Toeplitz methods for polynomial matrices proposed in [Zúñiga and Henrion, 2004a,b,c]. It is our opinion that research efforts must be devoted to problems with polynomials solved in a context of limited accuracy (scientific computing), see [Stetter, 2004]. The application of this conceptual framework to our algorithms has to be considered in the future.

In this paper we determined backward error bounds for the block Toeplitz algorithms of [Zúñiga and Henrion, 2004a] computing the eigenstructure of a polynomial matrix. These bounds are given by equations (6) and (10) for computing the null-space structure and the finite structure respectively. The obtained bounds are more pessimistic than the ideal ones given by equations (5) and (9). Sharper bounds can be expected from a componentwise error analysis [Higham, 2002]. The analysis with geometrical arguments as in [Edelman and Murakami, 1995] can also be considered as a future line of research. The objective of such analysis should be the derivation of exact first order expressions of the errors in the coefficients of a polynomial matrix due to the perturbations in the analyzed Toeplitz matrices. Along the lines proposed in [Lemonnier and Van Dooren, 2004] or [Fan et al., 2003], we can also consider the study of optimal scaling techniques over these Toeplitz matrices.

Ill-posedness of the rank revealing problem renders the problem of obtaining the eigenstructure of a polynomial matrix also ill-posed or $\infty$ conditioned. Ill-posed problems arise in several fields of scientific computing and thus, represent a challenge in numerical computations. As explained in [Stetter, 2004], a lot of meaningful illposed problems can be solved numerically very satisfactorily in practice. In [Zeng, 2004] some geometrical strategies allow to reformulate some illposed problems restoring the well-posedness and even making them well-conditioned. So, another line of future research could be the extension of these strategies to the polynomial eigenstructure problem.

\section{ACKNOWLEDGMENTS}

Juan-Carlos Zúñiga acknowledges support from the National Council of Science and Technology of Mexico (CONACYT) as well as from the Secretariat of Public Education of Mexico (SEP). Didier Henrion acknowledges support from project No. 102/02/0709 of the Grant Agency of the Czech Republic, as well as from project No. ME $698 / 2003$ of the Ministry of Education of the Czech Republic.

\section{REFERENCES}

Beelen Th. G. J. and Veltkamp G. W. (1987). Numerical computation of a coprime factorization of a transfer function matrix, Systems Control Lett., 9:281-288. 
Chu E. K. W. (2003). Perturbation of eigenvalues for matrix polynomials via the Bauer-Fike theorems. SIAM J. Matrix Anal. Appl., 25(2):551-573.

Edelman A. and Murakami H. (1995). Polynomial roots from companion matrix eigenvalues. Math. Comput., 64(210):763-776.

Fan H. Y., Lin W. W. and Van Dooren P. (2003). A note on optimal scaling of second order polynomial matrices. To appear in SIAM J. Matrix Anal. Appl.

Gantmacher F. R. (1959). Theory of Matrices I \& II, Chelsea, New York.

Golub G.H. and Van Loan C.F. (1996). Matrix Computations. The Johns Hopkins University Press, New York.

Henrion D. and Šebek M. (1999). Reliable numerical methods for polynomial matrix triangularization. IEEE Trans. Automat. Control, 4(3):497-508.

Higham N.J. (1990). Analysis of the Cholesky decomposition of a semi-definite matrix. Reliable Numerical Computations, Oxford University Press, 161-185.

Higham N.J. (2002). Accuracy and Stability of Numerical Algorithms. SIAM, Philadelphia.

Kailath T. (1980). Linear Systems. Prentice Hall, Englewood Cliffs.

Kučera V. (1993). Diophantine equations in control, a survey. Automatica, 29(6):1361-1375

Lemonnier D. and Van Dooren P. (2004). Optimal scaling of block companion pencils. Proceedings of the International Symposium on Mathematical Theory of Networks and Systems, Leuven, Belgium.

Moler C. and Stewart G. W. (1973). An algorithm for generalized matrix eigenvalue problems. SIAM J. Numer. Anal., 10:241-256.

Stefanidis P., Papliński A. P. and Gibbard M. J. (1992). Numerical operations with polynomial matrices: Application to multi-variable dynamic compensator design. Lecture Notes in Control and Inform. Sci., 171, Springer Verlag, New York.

Stetter H. J. (2004). Numerical Polynomial Algebra. SIAM, Philadelphia.

Tisseur F. and Meerbergen K. (2001). The quadratic eigenvalue problem. SIAM Review, 43:235-286.

Van Dooren P. and Dewilde P. (1983). The Eigenstructure of an Arbitrary Polynomial Matrix. Computational Aspects. Linear Algebra Appl., 50:545-580.

Zeng Z. (2004). Computing multiple roots of inexact polynomials. To appear in Math. Comput.

Zúñiga J.C. and Henrion D. (2004a). Block Toeplitz Methods in Polynomial Matrix Computations. Proceedings of the International Symposium on Mathematical Theory of Networks and Systems, Leuven, Belgium.

Zúñiga J.C. and Henrion D. (2004b). A Toeplitz algorithm for polynomial $J$-spectral factorization. Proceedings of the IFAC Symposium on System, Structure and Control, Oaxaca, Mexico.

Zúñiga J.C. and Henrion D. (2004c). On the application of displacement structure methods to obtain null-spaces of polynomial matrices. Proceedings of the IEEE Conference on Decision and Control, Paradise Island, Bahamas. 